\section{Inflammation and lipid profile in rheumatoid arthritis: bridging an apparent paradox}

\author{
Miguel A González-Gay, ${ }^{1}$ Carlos González-Juanatey ${ }^{2}$
}

Several pieces of evidence indicate that rheumatoid arthritis (RA) is a proatherogenic disease associated with increased cardiovascular (CV) mortality. ${ }^{1}{ }^{2}$ Besides genetic and traditional CV risk factors, ${ }^{3} 4$ chronic inflammation has emerged as a pivotal component implicated in the development of this process. A chronic inflammatory burden, determined by the mean values of C-reactive protein (CRP) over the extended follow-up of patients with RA, was associated with subclinical atherosclerosis and increased risk of $\mathrm{CV}$ events. ${ }^{5} 6$ In keeping with these observations, in a large retrospective cohort study, CV disease-related mortality was increased in RA patients with elevated measures of inflammation markers. ${ }^{7}$ These findings are in accordance with data from the general population that showed that CRP could be an independent predictor of $\mathrm{CV}$ disease with CRP values $\geq 3 \mathrm{mg} / \mathrm{L}$ defining high CV-risk individuals. ${ }^{8}$ With respect to this, levels of the proinflammatory cytokine interleukin-6, the main inducer of CRP, also predict outcome following hospitalisation for unstable angina. ${ }^{9}$

Despite some similarities, there are also some differences between patients with chronic inflammatory diseases and the general population. While the risk to develop atherosclerosis increases progressively with increasing low-density lipoprotein (LDL) cholesterol levels and declines with increasing levels of high-density lipoprotein (HDL) cholesterol in healthy individuals, ${ }^{10}$ the presence of a proinflammatory state leads to a decrease of total cholesterol, HDL cholesterol and LDL cholesterol in patients with RA. ${ }^{11}$

Paradoxically, anti-inflammatory therapies increase total cholesterol, HDL cholesterol and LDL cholesterol to variable degrees in patients with RA. ${ }^{11}{ }^{12}$ This

\footnotetext{
${ }^{1}$ Division of Rheumatology, Hospital Universitario Marqués de Valdecilla, IFIMAV, Santander, Cantabria, Spain; ${ }^{2}$ Division of Cardiology, Hospital Universitario Lucus Augusti, Lugo, Spain

Correspondence to Dr Miguel A González-Gay, Rheumatology Division, Hospital Universitario Marqués de Valdecilla, IFIMAV, Avenida de Valdecilla, s/n, Santander, Cantabria 39008, Spain; miguelaggay@ hotmail.com
}

lipid increase is not associated with a rise in the number of $\mathrm{CV}$ events in these patients. On the contrary, probably due to the anti-inflammatory effect, conventional disease-modifying antirheumatic drugs, such as methotrexate, and different biological agents have demonstrated a reduction of the $\mathrm{CV}$ death rate in patients with RA. ${ }^{13-16}$ A good example of this was the information retrieved from the British Society for Rheumatology Biologics Register that unveiled a reduction in the rate of myocardial infarction in patients with RA who were responders to biological agents. ${ }^{16}$ This reduction in the rate of CV events seems to be directly related to better control of the rheumatic disease. Therefore, the relationship between CV disease and lipid levels in patients with RA may be different from that observed in the general population.

Chronic inflammation leads to oxidative changes that alter HDL structure and reduce apolipoprotein-A-I in patients with active RA. ${ }^{17}$ Levels of paraoxonase-1, an antioxidant enzyme associated with HDL, are lower in patients with RA compared with healthy controls. ${ }^{18}$ Therefore, because of inflammation there is an impairment of the normal antiinflammatory, antioxidate and cardioprotective function of HDL cholesterol that turns out to be proinflammatory. However, the level and function of HDL is a very difficult part of lipidology and the relationship to $\mathrm{CV}$ disease is complex and poorly understood. In addition, studies on the levels and function of HDL cholesterol in the development of $\mathrm{CV}$ disease have often been done with murine macrophages and with concentrations of lipids out of physiological ranges. These evidences constitute a limitation for the interpretation of the results derived from these studies. In this regard, recent studies have shown that there is a lack of beneficial effect on $\mathrm{CV}$ disease risk of cholesteryl ester transfer protein (CETP) inhibitors, which inhibit reverse cholesterol transport and thus increase HDL levels, suggesting that although HDL cholesterol is a valuable biomarker in atherosclerosis, it might not be a preventative agent. ${ }^{19}$ Therefore, although we cannot negate the potential for HDL particles modified as a result of inflammatory processes to be causally related to atherogenesis, further studies are needed to examine this issue. With respect to this, regardless of the possible increase of lipid levels, the use of new biological therapies in patients with RA has beneficial qualitative effect on HDL cholesterol. This fact was highlighted by Popa et $a l^{20}$ who described changes in the HDL antioxidative capacity expressed by an increase in paraoxonase activity following antitumour necrosis factor therapy in patients with RA.

As discussed above, a reduction in lipid levels due to active disease does not imply a decrease of $\mathrm{CV}$ risk in patients with RA. With respect to this, in the Apolipoprotein MOrtality RISk (AMORIS) study that included 480406 people, with a mean follow-up of almost 12 years, including 1779 patients with RA of whom 214 had an acute myocardial infarction and 165 an ischaemic stroke, the levels of total cholesterol were significantly lower in patients with RA than in people without RA. ${ }^{21}$ However, despite these lower levels of total cholesterol, the rate of acute myocardial infarction and ischaemic stroke per 1000 years was at least 1.6 times higher in RA than in non-RA. ${ }^{21}$ In accordance with these observations, Myasoedova et al ${ }^{7}$ found a significant nonlinear association of total cholesterol with the risk of $\mathrm{CV}$ disease in RA, with 3.3-fold increased risk when total cholesterol values were $<4 \mathrm{mmol} / \mathrm{L} \quad(<155 \mathrm{mg} / \mathrm{dL})$ and no increased risk of $\mathrm{CV}$ disease when total cholesterol was $\geq 4 \mathrm{mmol} / \mathrm{L}$. These investigators suggested that, in contrast to the linear logarithmic association seen in individuals without inflammatory rheumatic diseases, $\mathrm{CV}$ disease risk in relation to total cholesterol and LDL cholesterol levels could be potentially represented by a U-shaped curve in patients with RA. This pattern explains the increased CV disease risk observed in patients with RA with active disease despite reduced serum cholesterol levels compared with the general population. ${ }^{7}$ In line with these findings, a recent study that assessed a retrospective cohort using 2005-2010 data from a large US commercial health plan supported the association of inflammatory markers and serum lipids with the risk of CV events in RA. ${ }^{22}$ The authors of this study implied that inflammatory markers and HDL cholesterol levels are associated with increased and reduced incident $\mathrm{CV}$ disease, respectively. They also provided further information on the controversial relationship between LDL cholesterol and CV disease 
in patients with RA. ${ }^{22}$ According to their results, higher CRP and erythrocyte sedimentation rate levels and lower HDL cholesterol levels were associated with higher incidence of myocardial infarction. ${ }^{22}$ Interestingly, HDL cholesterol $\geq 60 \mathrm{mg} / \mathrm{dL}$ compared with $<40 \mathrm{mg} / \mathrm{dL}$ was significantly associated with reduced risk of myocardial infarction. ${ }^{22}$ Another important result derived from this study was a nonlinear association between LDL cholesterol and myocardial infarction; the lowest risk was observed among patients with LDL cholesterol between 70 and $130 \mathrm{mg} / \mathrm{L}^{22}$

Taken together, the results reported by Zhang et $a l^{22}$ support the relevance of inflammation in the risk of $\mathrm{CV}$ disease in RA. They also indicate that the protective effect mediated by HDL cholesterol and found in the general population is also reproducible in patients with RA. On the other hand, this apparently paradoxical association of LDL cholesterol with CV mortality in RA resembles that reported in patients with advanced diseases associated with malnutrition, inflammation and cachexia such as in patients with chronic kidney disease that are not yet on dialysis. ${ }^{23}$ However, a limitation in the study by Zhang et $a l^{22}$ is that the cohort was mainly composed of males and RA is predominantly a female disease. It should be kept in mind as it well known that there are several gender differences relating to CV disease.

Provan et $a l^{24}$ confirmed that RA patients with $\mathrm{CV}$ disease have significantly increased levels of CV disease risk markers such as higher levels of NT-proBNP, brachial systolic pressure, augmentation index and central systolic pressure, but lower cholesterol than patients in remission and controls. They also have significantly higher levels of pulse wave velocity than those patients with RA who are in remission. $^{24}$ In addition, a recent study has demonstrated that patients with RA with active disease have higher vulnerability of carotid plaques than those in clinical remission. $^{25}$ Taken together; these extremely informative observations emphasise the need of achieving disease remission in patients with RA. Therefore, in the light of these results one may wonder what may be the best strategy to reduce the risk of $\mathrm{CV}$ events in patients with RA. Besides the use of therapies aimed to achieve disease remission, rheumatologists should follow national CV risk guidelines for controlling lipid levels and managing CV risk. This procedure should be conducted within the strategies aimed to achieve an adequate stratification of the $\mathrm{CV}$ risk in these patients. However, since
RA is a complex disease, adequate stratification of the $\mathrm{CV}$ risk in patients with RA is still far from being completely established. Interestingly, the European League Against Rheumatism (EULAR) task force recommended the use of the atherogenic index (total cholesterol/HDL cholesterol) rather than total cholesterol in the stratification of the CV risk of patients with RA. ${ }^{26}$ This proposal minimises the expected increase of total cholesterol associated with reduction of inflammation following tight control of the disease. However, despite efforts to adapt classic risk assessment tools used to estimate the $\mathrm{CV}$ risk in the general population, different reports have shown that well-established risk charts used for CV estimates, such as the Systematic Coronary Risk Evaluation (SCORE), which was modified according to the EULAR task force recommendations, ${ }^{26}$ often underestimate the actual CV risk of patients with RA. ${ }^{27}$ Data from recent reports highlight this fact showing that carotid ultrasound may be a useful tool to establish severe atherosclerotic disease in some patients with RA considered as having moderate $\mathrm{CV}$ disease risk when the modified EULAR SCORE was applied. ${ }^{28}{ }^{29}$ It has great relevance as the latest European guidelines categorise patients with carotid atherosclerotic plaques into very high $\mathrm{CV}$ risk equal to those with coronary heart disease. ${ }^{10}$ Patients with RA and carotid plaques must receive intensive lipid-lowering therapy equivalent to those with coronary heart disease.

In practical terms, the use of lipidlowering drugs should be kept in mind by clinicians in charge of the management of RA. Lipid levels should be periodically monitored, particularly when antirheumatic agent therapy is intensified. The inverse relationship between inflammation and lipid levels together with the adverse qualitative influence of inflammation on HDL cholesterol in the presence of heightened $\mathrm{CV}$ risk in RA could be expected to translate into adequate LDL cholesterol targets being lower in RA compared with in non-RA subjects. However, the question on to whom to treat and when to initiate statin treatment is not fully established in patients with RA. At this stage, a practical way of approaching this issue is to check the European guidelines on CV disease prevention in the clinical practice recommendations. ${ }^{10}$ According to these guidelines, there is not enough scientific evidence for any HDL cholesterol value to be considered as a goal of therapy, although HDL cholesterol $<1.0 \mathrm{mmol} / \mathrm{L}(<40 \mathrm{mg} / \mathrm{dL})$ in men and $<1.2 \mathrm{mmol} / \mathrm{L} \quad(<45 \mathrm{mg} / \mathrm{dL})$ in women should be considered as a marker of increased $\mathrm{CV}$ risk. ${ }^{10}$ With regard to LDL cholesterol, the European guidelines establish different risk categories and a series of target levels. In subjects at moderate risk (SCORE level $\geq 1$ to $<5 \%$ ), they recommend achieving LDL cholesterol goal $<3.0 \mathrm{mmol} / \mathrm{L}(<115 \mathrm{mg} / \mathrm{dL})$. In patients at high CV risk (SCORE level $\geq 5$ to $<10 \%$ ), a LDL cholesterol goal $<2.5 \mathrm{mmol} / \mathrm{L}$ $(<100 \mathrm{mg} / \mathrm{dL})$ is recommended. In those at very high $\mathrm{CV}$ disease risk (SCORE $\geq 10 \%$ ), the recommended LDL cholesterol target is $<1.8 \mathrm{mmol} / \mathrm{L} \quad(<70 \mathrm{mg} / \mathrm{dL})$ or a $\geq 50 \%$ LDL cholesterol reduction when the target level cannot be reached. We feel that it may also be applicable to patients with RA. However, as emphasised before, we have to take into account that in patients with moderate $\mathrm{CV}$ risk that are reclassified as having very high $\mathrm{CV}$ risk by a non-invasive technique (eg, because of the presence of plaques in the carotid ultrasound) LDL cholesterol target should be similar to that for individuals with SCORE $\geq 10 \%$.

Statin therapy has a beneficial effect on atherosclerotic CV disease outcomes in the general population. Once a practical procedure for the management of lipids in patients with RA to reach specific LDL cholesterol targets is established, we may wonder whether statins are as effective in RA patients as reported in the general population since inflammation is associated with changes in the lipid profile of patients with RA. Answer to this question was provided by Norwegian investigators. ${ }^{30} 31$ Semb et $a l^{30}$ showed that intensive treatment with statins led to a comparable decrease in lipid levels and a $20 \%$ reduction in overall risk of CV disease in patients with and without inflammatory joint disease. More recently, Rollefstad et $a l^{31}$ have confirmed that lipid lowering with statins in patients with $\mathrm{RA}$ is as effective as in the general population in less than three consultations and with few side effects. Further evidences support this beneficial effects since statins improve endothelial function that is impaired in patients with RA. ${ }^{32} 33$ A significant reduction of CRP levels in patients with RA taking statins compared with placebo has also been observed. ${ }^{34}$ In a randomised controlled trial of atorvastatin in RA, McCarey et $a l^{34}$ demonstrated a moderate decrease in disease activity and a significant reduction in total cholesterol and LDL cholesterol in statin-treated patients with RA. All these evidences highlight the beneficial effect of these drugs on the CV risk of these patients.

In conclusion, despite the apparent paradox between the lipid profile and 
inflammation, clinicians should keep in mind that statins have to be considered in the management of patients with RA.

Contributors MAG-G and CG-J contributed equally to the work.

Competing interests MAG-G's studies have been supported by grants from 'Fondo de Investigaciones Sanitarias' PI06/0024, PS09/00748 and PI12/00060, and RD12/0009/0013 (RIER) from 'Instituto de Salud Carlos III' (ISCIII) (Spain).

Provenance and peer review Commissioned; externally peer reviewed.

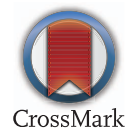

To cite González-Gay MA, González-Juanatey C. Ann Rheum Dis 2014:73:1281-1283.

Received 2 December 2013

Revised 4 January 2014

Accepted 24 January 2014

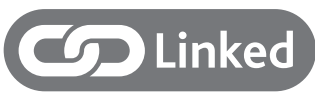

http://dx.doi.org/10.1136/annrheumdis-2013204715

Ann Rheum Dis 2014;73:1281-1283.

doi:10.1136/annrheumdis-2013-204933

\section{REFERENCES}

1 Gonzalez-Gay MA, Gonzalez-Juanatey C, Martin J. Rheumatoid arthritis: a disease associated with accelerated atherogenesis. Semin Arthritis Rheum 2005;35:8-17.

2 Aviña-Zubieta JA, Choi HK, Sadatsafavi M, et al. Risk of cardiovascular mortality in patients with rheumatoid arthritis: a meta-analysis of observational studies. Arthritis Rheum 2008;59:1690-7.

3 Dessein PH, Joffe BI, Veller MG, et al. Traditional and nontraditional cardiovascular risk factors are associated with atherosclerosis in rheumatoid arthritis. I Rheumatol 2005:32:435-42.

4 López-Mejías R, García-Bermúdez M, González-Juanatey C, et al. NFKB1-94ATTG ins/del polymorphism (rs28362491) is associated with cardiovascular disease in patients with rheumatoid arthritis. Atherosclerosis 2012;224:426-9.

5 Gonzalez-Gay MA, Gonzalez-Juanatey C, Piñeiro A, et al. High-grade C-reactive protein elevation correlates with accelerated atherogenesis in patients with rheumatoid arthritis. J Rheumatol 2005:32:1219-23.

6 Gonzalez-Gay MA, Gonzalez-Juanatey C, LopezDiaz MJ, et al. HLA-DRB1 and persistent chronic inflammation contribute to cardiovascular events and cardiovascular mortality in patients with rheumatoid arthritis. Arthritis Rheum 2007;57:125-32.

7 Myasoedova E, Crowson CS, Kremers HM, et al. Lipid paradox in rheumatoid arthritis: the impact of serum lipid measures and systemic inflammation on the risk of cardiovascular disease. Ann Rheum Dis 2011;70:482-7.

8 Ridker PM, Cook N. Clinical usefulness of very high and very low levels of $\mathrm{C}$-reactive protein across the full range of Framingham Risk Scores. Circulation 2004;109:1955-9.

9 Biasucci LM, Liuzzo G, Fantuzzi G, et al. Increasing levels of interleukin (IL)-1Ra and IL-6 during the first 2 days of hospitalization in unstable angina are associated with increased risk of in-hospital coronary events. Circulation 1999:99:2079-84.

10 Perk J, De Backer G, Gohlke H, et al. European Guidelines on cardiovascular disease prevention in clinical practice (version 2012). The Fifth Joint Task Force of the European Society of Cardiology and Other Societies on Cardiovascular Disease Prevention in Clinical Practice (constituted by representatives of nine societies and by invited experts). Eur Heart $J$ 2012;33:1635-701.

11 Robertson J, Peters MJ, Mclnnes IB, et al. Changes in lipid levels with inflammation and therapy in RA: a maturing paradigm. Nat Rev Rheumatol 2013;9:513-23.

12 van Sijl AM, Peters MJ, Knol DL, et al. The effect of TNF-alpha blocking therapy on lipid levels in rheumatoid arthritis: a meta-analysis. Semin Arthritis Rheum 2011:41:393-400.

13 Choi HK, Hernán MA, Seeger JD, et al. Methotrexate and mortality in patients with rheumatoid arthritis: a prospective study. Lancet 2002:359:1173-7.

14 Greenberg JD, Kremer JM, Curtis JR, et al. Tumour necrosis factor antagonist use and associated risk reduction of cardiovascular events among patients with rheumatoid arthritis. Ann Rheum Dis 2011;70:576-82.

15 Barnabe C, Martin BJ, Ghali WA. Systematic review and meta-analysis: anti-tumor necrosis factor $\alpha$ therapy and cardiovascular events in rheumatoid arthritis. Arthritis Care Res (Hoboken) 2011:63: 522-9.

16 Dixon WG, Watson KD, Lunt M, et al. Reduction in the incidence of myocardial infarction in patients with rheumatoid arthritis who respond to anti-tumor necrosis factor alpha therapy: results from the British Society for Rheumatology Biologics Register. Arthritis Rheum 2007;56:2905-12.

17 Charles-Schoeman C, Watanabe J, Lee YY, et al. Abnormal function of high-density lipoprotein is associated with poor disease control and an altered protein cargo in rheumatoid arthritis. Arthritis Rheum 2009;60:2870-9

18 Charles-Schoeman C, Lee YY, Grijalva V, et al. Cholesterol efflux by high density lipoproteins is impaired in patients with active rheumatoid arthritis. Ann Rheum Dis 2012;71:1157-62.

19 Barter PJ, Rye KA. Cholesteryl ester transfer protein inhibition as a strategy to reduce cardiovascular risk. J Lipid Res 2012;53:1755-66.

20 Popa C, van Tits LJ, Barrera P, et al. Anti-inflammatory therapy with tumour necrosis factor alpha inhibitors improves high-density lipoprotein cholesterol antioxidative capacity in rheumatoid arthritis patients. Ann Rheum Dis 2009;68:868-72.
21 Semb AG, Kvien TK, Aastveit AH, et al. Lipids, myocardial infarction and ischaemic stroke in patients with rheumatoid arthritis in the Apolipoprotein-related Mortality RISk (AMORIS) Study. Ann Rheum Dis 2010;69:1996-2001.

22 Zhang J, Chen L, Delzell E, et al. The association between inflammatory markers, serum lipids and the risk of cardiovascular events in patients with rheumatoid arthritis. Ann Rheum Dis 2014;73: 1301-8

23 Kovesdy CP, Anderson JE, Kalantar-Zadeh K. Inverse association between lipid levels and mortality in men with chronic kidney disease who are not yet on dialysis: effects of case mix and the malnutrition inflammation-cachexia syndrome. J Am Soc Nephrol 2007;18:304-11.

24 Provan SA, Semb AG, Hisdal J, et al. Remission is the goal for cardiovascular risk management in patients with rheumatoid arthritis: a cross-sectional comparative study. Ann Rheum Dis 2011;70: 812-17.

25 Semb AG, Rollefstad S, Provan SA, et al. Carotid plaque characteristics and disease activity in rheumatoid arthritis. J Rheumatol 2013;40: 359-68.

26 Peters MJ, Symmons DP, McCarey D, et al. EULAR evidence-based recommendations for cardiovascular risk management in patients with rheumatoid arthritis and other forms of inflammatory arthritis. Ann Rheum Dis 2010:69:325-31.

27 Gómez-Vaquero C, Corrales A, Zacarías A, et al. SCORE and REGICOR function charts underestimate the cardiovascular risk in Spanish patients with rheumatoid arthritis. Arthritis Res Ther 2013:15:R91.

28 Corrales A, González-Juanatey C, Peiró ME, et al. Carotid ultrasound is useful for the cardiovascular risk stratification of patients with rheumatoid arthritis: results of a population-based study. Ann Rheum Dis 2014:73:722-7.

29 Corrales A, Parra JA, González-Juanatey C, et al. Cardiovascular risk stratification in rheumatic diseases: carotid ultrasound is more sensitive than Coronary Artery Calcification Score to detect subclinical atherosclerosis in patients with rheumatoid arthritis. Ann Rheum Dis 2013:72:1764-70.

30 Semb AG, Kvien TK, DeMicco DA, et al. Effect of intensive lipid-lowering therapy on cardiovascular outcome in patients with and those without inflammatory joint disease. Arthritis Rheum 2012:64:2836-46.

31 Rollefstad S, Kvien TK, Holme I, et al. Treatment to lipid targets in patients with inflammatory joint diseases in a preventive cardio-rheuma clinic. Ann Rheum Dis 2013:72:1968-74.

32 Hermann F, Forster A, Chenevard R, et al. Simvastatin improves endothelial function in patients with rheumatoid arthritis. I Am Coll Cardiol 2005:45:461-4.

33 Gonzalez-Juanatey C, Testa A, Garcia-Castelo A, et al. HLA-DRB1 status affects endothelial function in treated patients with rheumatoid arthritis. Am J Med 2003;114:647-52.

34 McCarey DW, McInnes IB, Madhok R, et al. Trial of Atorvastatin in Rheumatoid Arthritis (TARA): double-blind, randomized placebo-controlled trial. Lancet 2004;363:2015-21. 